\title{
Genotyping of the Human Lipoprotein Lipase Gene by Ferrocenylnaphthalene Diimide-based Electrochemical Hybridization Assay
}

\author{
Takahiko NoJIMA, ${ }^{* 1}$ Kenichi Yamashita, ${ }^{* 1}$ Atsuko TaKagI, ${ }^{2}$ Yasuyuki IKeda, ${ }^{* 3}$ Hiroki Kondo, ${ }^{* 4}$ \\ and Shigeori TAKENAKA*5† \\ *1 Department of Applied Chemistry, Faculty of Engineering, Kyushu University, Fukuoka 819-0395, Japan \\ *2 Department of Etiology and Pathophysiology, National Cardiovascular Center Research Institute, \\ Osaka 565-8565, Japan \\ *3 Department of Pharmacology, National Cardiovascular Center Research Institute, Osaka 565-8565, Japan \\ *4 Department of Biochemical Engineering and Science, Kyushu Institute of Technology, Iizuka 820-8502, Japan \\ *5 Department of Materials Science, Faculty of Engineering, Kyushu Institute of Technology, \\ Kitakyushu 804-8550, Japan
}

\begin{abstract}
A ferrocenylnaphthalene diimide (FND)-based electrochemical hybridization assay (FND-EHA) was applied to the detection of two mutations in human lipoprotein lipase (LPL) gene, G188E (one base transition) and Arita (one base deletion). A probe oligodeoxyribonucleotide of 13 bases representing the wild type (WT) sequence of LPL was immobilized on a gold electrode, followed by hybridization with a sample PCR product of 350 base pairs under conditions in which both WT and mutated (MT) sequences could form a duplex with the probe. The hybridized electrodes were soaked in an electrolyte containing FND under conditions in which only the mismatched duplex could undergo dissociation. FND was concentrated in proportion to the amount of the duplex remaining on the electrode to give rise to a current signal. Blind tests were run to judge the genotype (WT/WT, WT/MT, or MT/MT) of 10 samples each for the G188E and Arita mutations and then, 8 and 10 of them were judged correctly, respectively.
\end{abstract}

(Received August 2, 2005; Accepted October 13, 2005)

\section{Introduction}

The development of the detection methods for single nucleotide polymorphisms (SNPs) is desired to realize a genome-based tailor-made diagnosis. ${ }^{1}$ SNP is detected by hybridization of probe DNA representing the SNP-containing region with sample DNA. A one-base mismatched duplex is formed upon hybridization, which is distinguished from a fully matched duplex. SNPs can be detected by using several DNA probes carrying different SNPs. Such methods have been utilized for routine SNP detection. ${ }^{2}$

Aiming at a simple and high-speed detection method, we developed an electrochemical hybridization assay (EHA) based on ferrocenylnaphthalenediimide (FND) as an electrochemical indicator (FND-EHA), ${ }^{3}$ and applied it for the detection of onebase mismatches. ${ }^{4}$ Since the autosomal chromosome of humans has a pair of alleles, heterozygous genes may be discriminated from homozygotes from the quantity of the allele in question, one half that of the latter. We developed a discrimination method for the SNP in heterozygotes by using two kinds of electrodes carrying two DNA probes representing wild type (WT) or mutated (MT) sequences. ${ }^{5}$ Electrochemical signals were obtained in the presence of FND upon the formation of a duplex from the two electrodes, since heterozygotes give rise to

\footnotetext{
† To whom correspondence should be addressed.

E-mail: shige@che.kyutech.ac.jp
}

an equal magnitude of signals.

In order to simplify the system, we examined the detection of heterozygotes with a single electrode. ${ }^{6}$ The principle of this simplified version is shown in Fig. 1. Hybridization was performed under conditions in which both WT and MT could form a duplex with the probe DNA; the amount of duplex formed with heterozygous samples was the same as that with homozygotes. The electrochemical response was then measured under conditions in which the mismatch duplex was unstable to undergo dissociation. The response was to be decreased to one half, since the mismatched duplex carrying the SNP had been dissociated. Heterozygotes were discriminated irrespective of the concentration of sample DNA by using such a method. In the present report, the diagnosis of SNP in heterozygotes using one electrode is discussed concerning the human lipoprotein lipase (LPL) G188E mutant (G-to-A transition) ${ }^{7}$ and Arita mutant (one base deletion). ${ }^{8}$ The reliability of the technique was examined by blind tests for 10 unknown samples for each type of SNPs.

\section{Experimental}

\section{Materials}

The oligodeoxyribonucleotides were custom synthesized by Hokkaido System Science (Sapporo, Japan). Organization of the human lipoprotein lipase gene is illustrated in Fig. 1 with a focus on the region carrying G188E and Arita in exon 5, site 1 
(C)

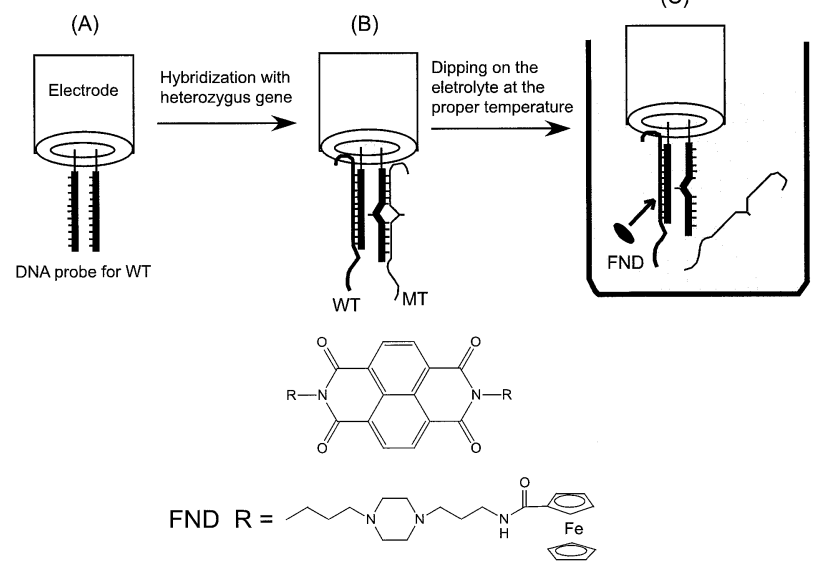

Fig. 1 Mechanism for the generation of an electrochemical signal for the heterozygote of an intensity half that of the homozygote in the FND-EHA method. (A) A DNA probe for WT is immobilized on the electrode. (B) A heterozygous DNA sample is allowed to hybridize on this electrode under conditions where a duplex is formed. (C) When the hybridized electrode is dipped in an electrolyte, the hybridized DNA for MT is easily dissociated from the electrode by selecting a proper condition. FND remains on the DNA duplex region, and hence the electrochemical signal of FND is half that for the homozygous WT gene without any stringent.

G188 WT or G188E MT and site 2 WT or Arita MT. The G188E mutation is a $\mathrm{G} \rightarrow \mathrm{A}$ transition $\left({ }^{818} \mathrm{G} \rightarrow \mathrm{A}\right)$, which results in Gly $\rightarrow$ Ala conversion. The Arita mutation involves the deletion of $916 \mathrm{G}$, which results in a frame-shift downstream of the deletion site. Nucleotide numbering (818 and 916) is based on that of the cDNA reported by Wion et al. ${ }^{14}$ Probe DNAs for site 1 G188 WT and site 2 WT have nucleotide sequences complementary to the 13 bases shown in Fig. 1. A thiol group was introduced on the $5^{\prime}$-teminus of the probes in order to immobilize them on the gold surface. Human chromosomal DNA was isolated from healthy male (YI, WT/WT), patients having a hetero LPL Arita allele (EN, WT/Arita), homo LPL Arita allele (TN, Arita/Arita), or hetero G188E allele (MN, WT/G188E) with a QIAamp blood kit (Qiagen K. K., Tokyo, Japan) according to the manufacturer's protocol. A 350-bp DNA fragment containing LPL exon 5 and the flanking regions was amplified from the chromosomal DNA by a polymerase chain reaction $(\mathrm{PCR})^{9}$ with primers forward (No. 259, 5'-TGT AAA ACG ACG GCC AGT AAA TTT ACA AAT CTG TGT TCC TGC TTT TT-3') and reverse (No. 260, 5'-CAG GAA ACA GCT ATG ACC GAT AAG AGT CAC ATT TAA TTC GCT TCT A-3'), followed by purification with a QIAquick PCR purification kit (Qiagen) according to the manufacturer's protocol. The concentration of DNA was determined with a molar absorptivity of $6500 \mathrm{~cm}^{-1} \mathrm{M}^{-1}$ for one base pair at 260 nm. ${ }^{10}$ Saline sodium citrate buffer (SSC) was prepared according to a standard protocol. ${ }^{11}$ The composition for $20 \times$ $\mathrm{SSC}$ is $300 \mathrm{mM}$ sodium citrate and $3 \mathrm{M}$ sodium chloride. All of the reagents used were of the highest grade available.

Immobilization of probe DNA on the gold electrode

Probe DNA was immobilized on the surface of a gold electrode through a $5^{\prime}$-thiol group. The gold surface (BAS No. 2014, $2 \mathrm{~mm}^{2}$ ) was boiled in $2 \mathrm{M} \mathrm{NaOH}$ for $1 \mathrm{~h}$, washed with water, and dried. It was swirled in concentrated nitric acid for $30 \mathrm{~min}$ at room temperature, washed with water, and dried. One microliter of probe DNA was placed on the gold surface, which

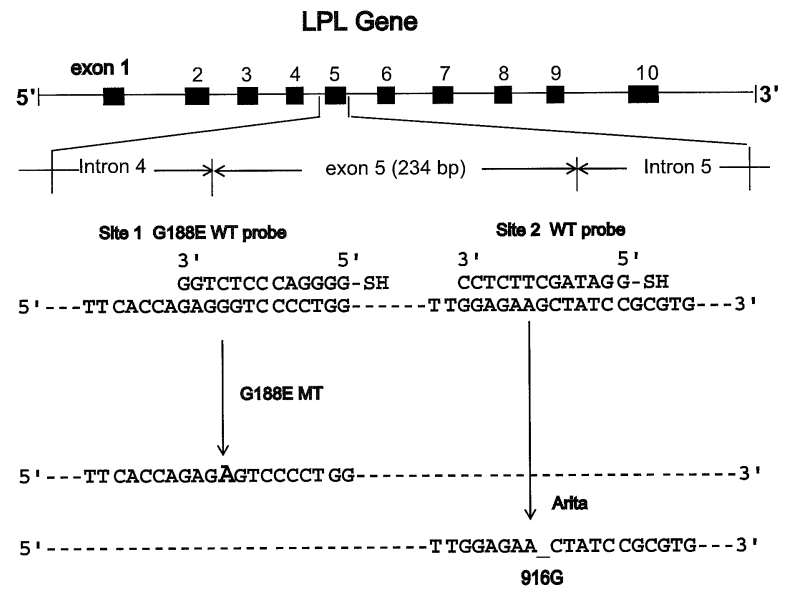

Fig. 2 Organization of the human lipoprotein lipase (LPL) gene with a focus on a region carrying G188E and Arita in exon 5, together with oligodeoxyribonucleotide probes (13 bases), site 1 G188 WT or G188E MT and site 2 WT and Arita MT. The G188E mutation is a $\mathrm{G} \rightarrow \mathrm{A}$ transition $\left({ }^{818} \mathrm{G} \rightarrow \mathrm{A}\right)$, which results in Gly $\rightarrow$ Ala conversion. The Arita mutation is the deletion of ${ }^{916} \mathrm{G}$, which results in a frame-shift. The nucleotide numbering is based on that of the cDNA reported by Wion et al. ${ }^{14}$

was then capped and incubated for $2 \mathrm{~h}$ at room temperature. The DNA concentrations used were 2 pmol $\mu \mathrm{L}^{-1}$ and $100 \mathrm{fmol}$ $\mu \mathrm{L}^{-1}$ for oligodeoxyribonucleotide and PCR samples, respectively. The electrode was then washed with water, dried, and $1 \mu \mathrm{L}$ of 2 -mercaptoethanol was added. After incubation for $2 \mathrm{~h}$ at room temperature, the electrode was washed with water and kept in water until use.

Temperature-dependency of duplex dissociation on the electrode One microliter of oligodeoxyribonucleotides of 13 bases (10 pmol $\mu \mathrm{L}^{-1}$ in $2 \times \mathrm{SSC}$ ) complementary to the probe sequence with (MT) and without one base mismatch (WT) was allowed to hybridize at room temperature for $1 \mathrm{~h}$ with the G188E WT probe-immobilized electrode prepared as mentioned above. The same experiment was carried out with the site $2 \mathrm{WT}$ probe. The electrode was then soaked in $2 \mathrm{~mL}$ of a measurement solution (0.1 M HOAc/KOAc, pH 5.6, $0.1 \mathrm{M} \mathrm{KCl}$, and $0.05 \mathrm{mM}$ FND). Differential pulse voltammetry (DPV) was determined at different temperatures. The conditions for the DPV measurements were as follows: initial potential, $0 \mathrm{~V}$; final potential, $0.6 \mathrm{~V}$; scan rate, $100 \mathrm{mV} \mathrm{s}^{-1}$; pulse amplitude, $0.05 \mathrm{~V}$; sample width, $16.7 \mathrm{~ms}$; pulse period, $0.2 \mathrm{~s}$; pulse width, $0.05 \mathrm{~s}$; quiet time, $2 \mathrm{~s}$; sensitivity, $10^{-6} \mathrm{~A} \mathrm{~V}^{-1}$. The counter electrode and reference electrode were $\mathrm{Pt}$ and $\mathrm{Ag} / \mathrm{AgCl}$, respectively.

\section{Optimization of experimental temperature for oligodeoxy- ribonucleotides}

The experimental temperature was optimized with oligodeoxyribonucleotides of 13 bases. DPV was measured with a gold electrode modified with probe DNA ( 2 pmol). After the electrode was washed with water, $1 \mu \mathrm{L}$ of sample DNA (10 pmol $\mu \mathrm{L}^{-1}, 2 \times \mathrm{SSC}$ ) was dropped on it, and hybridization was allowed to proceed for $1 \mathrm{~h}$ at room temperature. DPV was then measured again. The response currents at various temperatures were measured.

Optimization of the hybridization condition for PCR products

DPV was measured with a gold electrode modified with a DNA probe $(2 \mathrm{pmol})$ and the electrode was washed with water. 


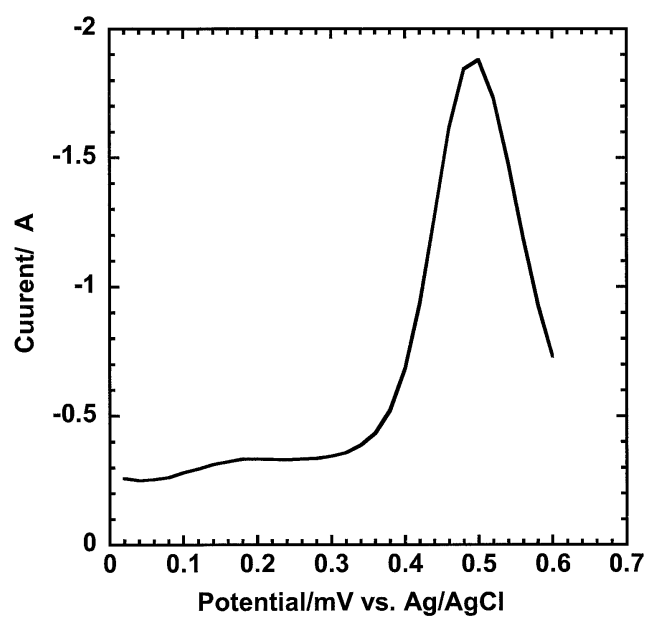

Fig. 3 DPV curves for the site 1 G188E WT probe on the electrode after hybridization of a WT sample in 0.1 M HOAc/KOAc ( $\mathrm{pH}$ 5.6) containing $0.1 \mathrm{M} \mathrm{KCl}$ and $0.05 \mathrm{mM} \mathrm{FND}$ at $25^{\circ} \mathrm{C}$.

One microliter of a PCR product $\left(5 \mathrm{pmol} \mu \mathrm{L}^{-1}\right)$ was heated at $98^{\circ} \mathrm{C}$ for $10 \mathrm{~min}$ and quickly cooled with liquid nitrogen. After the addition of various amounts of $20 \times$ SSC, hybridization was performed by standing the electrode at room temperature for 1 h. The response current was obtained by DPV measurement at several temperatures and several SSC concentrations.

\section{Blind test for SNP detection}

Ten unknown samples each of PCR products were prepared for G188E and Arita mutations, and electrochemical measurements were repeated 5 times for each sample under conditions identical to those described above. Based on the result, the genotype was judged.

\section{Results and Discussion}

\section{Duplex dissociation with a rise in temperature}

An oligodeoxyribonucleotide having a sequence complementary to a part of LPL exon 5 (Fig. 2) was immobilized on the surface of a gold electrode, with which a 13-base synthetic oligodeoxyribonucleotide having the WT or MT sequence was allowed to hybridize; DPV measured in the presence of $50 \mu \mathrm{M}$ FND at several temperatures where a duplex was formed. Typical DPV curves for an electrode having the DNA probe for WT in the case of G188E after hybridization with target DNA at $25^{\circ} \mathrm{C}$ are shown in Fig. 3. These currents decreased as the temperature was raised in both G188E and Arita detection, as shown in Fig. 4. The mismatched duplex started to dissociate at lower temperature because it was less stable in both cases.

\section{Optimization of the temperature for the detection of} oligodeoxyribonucleotides

In order to optimize the experimental temperature that gives the largest difference in peak currents upon duplex formation with WT or MT, peak currents were measured at various temperatures. It turned out that 40 and $33^{\circ} \mathrm{C}$ were the optimum temperatures for the site $1 \mathrm{G} 188$ and site 2 WT probes, respectively (Fig. 4). The difference in the peak current $(\Delta i \%)$ was defined as $\left(i-i_{0}\right) / i_{0} \times 100$, where $i_{0}$ and $i$ represent the peak current before and after hybridization, respectively. Index $\Delta i$ is the net current increase for FND, which bound to the duplex DNA formed from the DNA probe immobilized on the
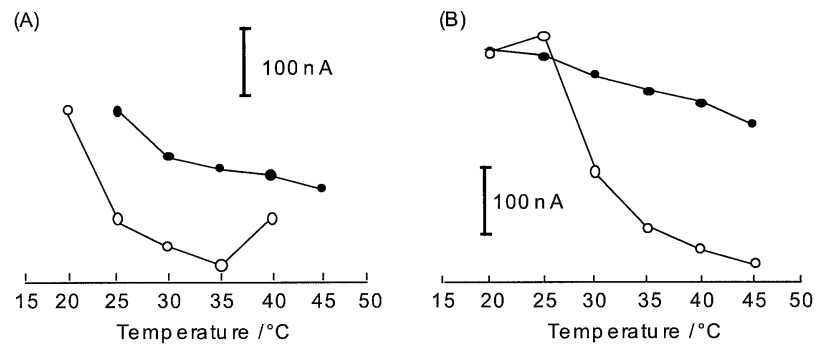

Fig. 4 Temperature-dependency of the peak current of $0.05 \mathrm{mM}$ FND. A duplex was formed from probe DNA of 13 bases G188 WT (A) or site 2 WT (B) and the target DNA of 13 bases representing the wild type (G188 WT and site 2 WT, closed circles) or mutant sequence (G188E MT and Arita MT, open circles) of LPL at different temperatures. The amount of the probe used for immobilization was 2 pmol and that of sample DNA 10 pmol.
(A)

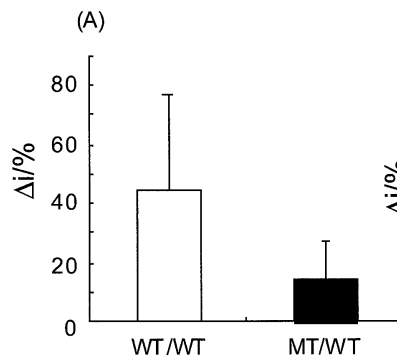

(B)

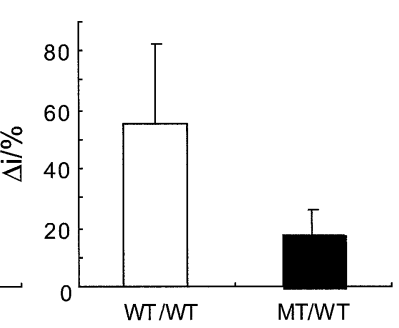

Fig. 5 Discrimination between wild- (WT) and mutant-type (MT) oligodeoxyribonucleotides of LPL by the electrochemical hybridization assay. (A) The site 1 G188 WT probe DNA of 13 bases was immobilized on a gold electrode, and its complementary DNA of the same length with (G188E MT) or without one base mismatch (G188 WT) was allowed to hybridize. The peak current due to FND before $\left(i_{\mathrm{o}}\right)$ and after hybridization $(i)$ was converted to $\Delta i$, which represents a net increase upon duplex formation. The $\Delta i$ was $44.9 \%$ with the site $1 \mathrm{G} 188$ WT sample, whereas it was $15.0 \%$ with the site $1 \mathrm{G} 188 \mathrm{E}$ MT. Error bars represent the average $\Delta i \pm$ standard deviations. (B) The same experiment as (A) with the site $2 \mathrm{WT}$ probe DNA. The complementary DNA of the same length without (site 2 WT) deletion or 12-mer with one base deletion (Arita MT) of LPL was allowed to hybridize. The $\Delta i$ was $55.6 \%$ with the site 2 WT sample, whereas it was $17.7 \%$ with the Arita MT.

electrode with sample DNA. As shown in Fig. 5A, the average current increase and mean standard error for the site $1 \mathrm{G} 188$ WT were 44.9 and $32.1 \%$, respectively, under these conditions, whereas those for site 1 G188E MT were 15.0 and $13.2 \%$. The corresponding values for site 2 WT were 55.6 and $26.9 \%$, and for site 2 Arita MT, 17.7 and 8.9\% (Fig. 5B).

Hybridization of the PCR product with immobilized probe DNA

A part of LPL exon 9 containing a mutation site was amplified by PCR. The obtained 350-bp DNA fragment was allowed to hybridize with the probe DNA immobilized on the gold electrode in $2 \times \mathrm{SSC}$ at room temperature for $1 \mathrm{~h}$. DPV was then measured at $40^{\circ} \mathrm{C}$ in $0.5 \times \mathrm{SSC}$ for G188E, or $33^{\circ} \mathrm{C}$ in 0.25 $\times$ SSC for Arita, and the data were converted to $\Delta i \%$. Because the amount of duplex formed at the surface of the electrode represents the abundance of the WT sequence in the PCR product, the ratio of $\Delta i \%$ for WT/WT and WT/MT should be 2:1. As shown in Fig. 6A, the current increase and the mean standard error for site 1 G188 WT were 78.9 and $32.6 \%$, 
respectively, whereas those for site 1 G188E MT were 45.7 and $14.1 \%$, which were close to the theoretical values. Likewise, the current increase and the mean standard error for site 2 WT/WT were 78.3 and $18.5 \%, 45.1$ and $14.8 \%$ for site 2 WT/Arita, and 25.0 and $11.8 \%$ for site 2 Arita/Arita, as shown in Fig. 6B. Although the standard errors were relatively large, the differences in $\Delta i \%$ among the three genotypes seemed to be large enough to allow their assignment.

\section{Blind test for unknown samples}

In order to run a blind test for unknown samples, a threshold was set to discriminate a hit from a miss as follows. It was defined as the sum of a mean minus the standard error for WT/WT and a mean plus standard error for WT/MT, divided by 2. Thus, based on the result shown in Fig. 6 for G188E, the threshold was $\{(78.9-32.6)+(45.7+14.1)\} / 2=53 \%$. Likewise, in the case of Arita detection, $\{(78.3-18.4)+(45.1+$
(A)

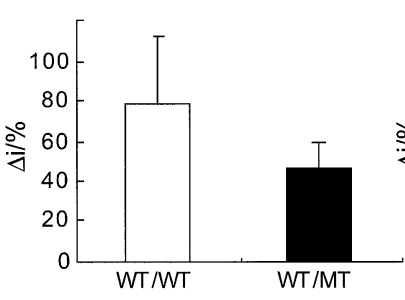

(B)

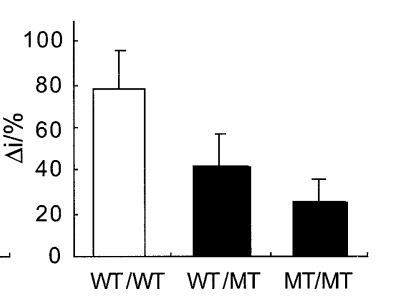

Fig. 6 Discrimination among homozygotes, WT/WT and MT/MT, and heterozygote WT/MT of LPL by the FND-EHA. The 350-bp PCR products, amplified from human chromosomal DNA, were analyzed with a gold electrode carrying the site $1 \mathrm{G} 188$ WT probe (A) or site $2 \mathrm{WT}$ probe (B) as in Fig. 3. (A) The $\Delta i$ values were $78.9 \%$ for WT/WT, and $45.7 \%$ for WT/MT. (B) The $\Delta i$ values were $78.3 \%$ for WT/WT, $45.1 \%$ for WT/Arita, and $25.0 \%$ for Arita/Arita. Error bars represent the average $\Delta i \pm$ standard deviations.
14.8) $\} / 2=60 \%$ was the threshold set to distinguish site 2 WT/Arita from site $2 \mathrm{WT} / \mathrm{WT}$. The threshold to distinguish the(del) site 2 WT/Arita from site 2 Arita/Arita was $\{(45.1$ $14.8)+(25.0+11.8)\} / 2=34 \%$. In other words, when the average of 5 measurements was over $60 \%$ in the blind test, the genotype is to be judged as site 2 WT/WT, between $60 \%$ and $34 \%$ as site 2 WT/Arita, and smaller than $34 \%$ as site 2 Arita/Arita.

Blind tests for G188E and Arita mutations were carried out. The measurement was repeated 5 times for 10 samples each, and the genotype was judged based on the threshold defined above. The results thus obtained are summarized in Fig. 7, where the genotype judged by this method is compared with that assigned by sequencing. For G188E detection, 8 of the 10 samples were judged correctly, whereas all of the 10 samples were judged properly for Arita mutation detection. The better score for the latter is explained by the higher stability of its duplex, as exemplified by the larger difference in the stability of mismatched duplex of one-base deletion than that of one-base transition (Fig. 4), which was favorable for the judgment.

\section{Conclusion}

We previously reported on the FND-based electrochemical genotyping of human LPL gene. This was achieved by using two electrodes carrying either a WT or MT sequence. To simplify the analysis, a one-electrode system was devised and its performance assessed herein. As described above, the overall performance of the new system in the detection of heterozygotes was comparable with that of the previous system. Yet, it is still not possible to discriminate heterozygotes from homozygotes in unknown samples perfectly, especially when a PCR, product was tested, presumably because its size is much larger than that of the probe and self-hybridization prevails. One way to overcome this problem may be a use of symmetric
(A)

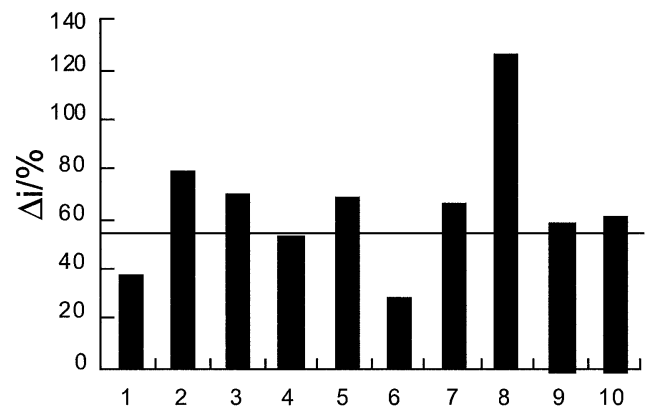

(B)

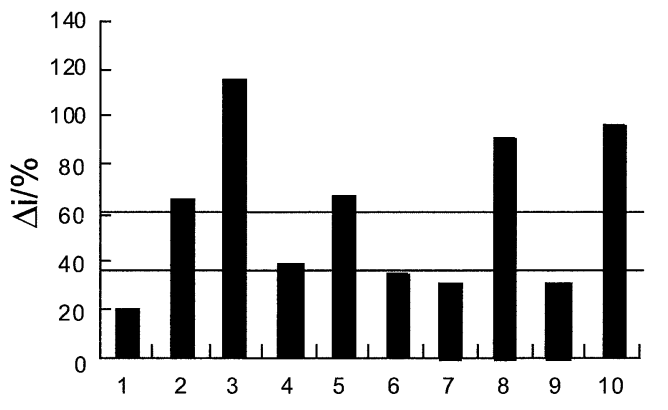

\begin{tabular}{ccccc}
\hline $\begin{array}{c}\text { Sample } \\
\text { number }\end{array}$ & & $\begin{array}{c}\text { Electrochemical } \\
\text { judgement }\end{array}$ & Sequence & $\begin{array}{c}\text { Right or } \\
\text { wrong }\end{array}$ \\
\hline 1 & 36.8 & WT/G188E & WT/G188E & 0 \\
2 & 79.3 & WT/WT & WT/WT & 0 \\
3 & 71.0 & WT/WT & WT/WT & 0 \\
4 & 52.2 & WT/G188E & WT/G188E & $\circ$ \\
5 & 69.7 & WT/WT & WT/WT & 0 \\
6 & 28.9 & WT/G188 & WT/WT & $\times$ \\
7 & 66.3 & WT/WT & WT/G188E & $\times$ \\
8 & 124.8 & WT/WT & WT/WT & 0 \\
9 & 58.5 & WT/WT & WT/WT & 0 \\
10 & 60.6 & WT/WT & WT/WT & 0 \\
\hline
\end{tabular}

\begin{tabular}{|c|c|c|c|c|}
\hline $\begin{array}{l}\text { Sample } \\
\text { number }\end{array}$ & $\begin{array}{c}\text { Average, } \\
\%\end{array}$ & $\begin{array}{c}\text { Electrochemical } \\
\text { judgement }\end{array}$ & Sequence & $\begin{array}{l}\text { Right or } \\
\text { wrong }\end{array}$ \\
\hline $\begin{array}{r}1 \\
2 \\
3 \\
4 \\
5 \\
6 \\
7 \\
8 \\
9 \\
10\end{array}$ & $\begin{array}{r}19.8 \\
66.7 \\
116.2 \\
38.4 \\
67.7 \\
34.5 \\
32.2 \\
91.1 \\
30.6 \\
97.0\end{array}$ & $\begin{array}{c}\text { Arita/Arita } \\
\text { WT/WT } \\
\text { WT/WT } \\
\text { WT/Arta } \\
\text { WT/WT } \\
\text { WT/Arta } \\
\text { Arta/Arta } \\
\text { WT/WT } \\
\text { Arta/Arta } \\
\text { WT/WT }\end{array}$ & $\begin{array}{c}\text { Arita/Arita } \\
\text { WT/WT } \\
\text { WT/WT } \\
\text { WT/Arita } \\
\text { WT/WT } \\
\text { WT/Arta } \\
\text { Arta/Arta } \\
\text { WT/WT } \\
\text { Arta/Arta } \\
\text { WT/WT }\end{array}$ & $\begin{array}{l}0 \\
0 \\
0 \\
0 \\
0 \\
0 \\
0 \\
0 \\
0 \\
0\end{array}$ \\
\hline
\end{tabular}

Fig. 7 Result of a blind test for the LPL site 1 G188E detection (A) and site 2 Arita detection (B). The electrochemical assay was repeated 5 times for each sample, and the genotype was judged from the magnitude of $\Delta i \%$ according to the criteria described in the text. 
PCR which can generate single-stranded DNA. Another reason for the failure lies in the variability in the performance of individual probe-immobilized electrodes, or in the amount of probe DNA immobilized on them. Unfortunately, this variability cannot be corrected, even by using $\Delta i$. Despite these problems, since the number of electrodes is cut to one half, this technique is especially advantageous when constructing a multielectrode system, such as an electrochemical array. Further studies are under way to attain this goal.

\section{Acknowledgements}

This work was supported in part by a Grant-in-Aid for Scientific Research from the Ministry of Education, Culture, Sports, Science and Technology (MEXT), Japan. The authors are also grateful for financial support from the Japan Society for the Promotion of Science (to K. Y.). This work was supported by the Takeda Science Foundation (to T. N.).

\section{References}

1. J. Brooks, Gene, 1999, 234, 177.

2. D. Y. Wu, G. Nozari, M. Schold, B. J. Conner, and R. B.
Wallace, DNA, 1989, 8, 135.

3. S. Takenaka, K. Yamashita, M. Takagi, Y. Uto, and H. Kondo, Anal. Chem., 2000, 72, 1334.

4. K. Yamashita, M. Takagi, H. Kondo, and S. Takenaka, Anal. Biochem., 2002, 306, 188.

5. K. Yamashita, A. Takagi, M. Takagi, H. Kondo, Y. Ikeda, and S. Takenaka, Bioconjugate Chem., 2002, 13, 1193.

6. T. Nojima, K. Yamashita, A. Takagi, M. Takagi, Y. Ikeda, H. Kondo, and S. Takenaka, Anal. Sci., 2003, 19, 79.

7. A. Takagi, Y. Ikeda, K. Tachi, T. Shinozuka, and A. Yamamoto, Clin. Chim. Acta, 1999, 285, 143.

8. M. Austin, J. E. Hokanson, and K. L. Edwards, Am. J. Cardiol., 1998, 81, 7B.

9. R. K. Saiki, D. H. Gelfand, S. Stoffel, S. J. Scharf, R. Higuchi, G. T. Horn, K. B. Mullis, and H. A. Erlich, Science, 1988, 239, 487

10. R. Cantor and M. M. Warchaw, Biopolymers, 1970, 9, 1059.

11. J. Sambrook, E. F. Fritsch, and T. Maniatis, "Molecular Cloning A Laboratory Manual", 2nd ed., 1989, Cold Spring Harbor Press, New York.

12. K. J. Livak, Gent. Anal., 1999, 14, 143.

13. L. A. Haff and I. P. Smirnov, Genome Res., 1997, 7, 378.

14. K. L. Wion, T. G. Kirchgessner, A. J. Lusis, M. C. Schotz, and R. M. Lawn, Science, 1987, 235, 1638. 